\title{
Sensitive Detection of Benzene in the Presence of Interferents by High Kinetic Energy lon Mobility Spectrometry
}

\author{
Ansgar T. Kirk, Jens Langejuergen, Maria Allers, Jens Oermann and Stefan Zimmermann \\ Leibniz University Hannover, Institute of Electrical Engineering and Measurement Technology, \\ Department of Sensors and Measurement Technology, Appelstr. 9A, 30167 Hannover, Germany \\ e-mail: kirk@geml.uni-hannover.de
}

\begin{abstract}
:
Ion mobility spectrometers (IMS) are widely used for fast trace gas detection in air, often achieving limits of detection in the pptv-range (parts per trillion) within one second averaging time due to the typically employed atmospheric pressure chemical ionization (APCI). However, IMS performance is highly dependent on the mixture of analytes present, as various quenching reactions can occur. For example, benzene, which is an important precursor in several processes but highly carcinogenic, is hard to detect under ambient conditions. Using an ion mobility spectrometer operated at high kinetic ion energies, we were able to detect $70 \mathrm{ppb}_{\mathrm{v}}$ benzene within two seconds averaging time even under humid conditions ( $90 \%$ r.H.) and in the presence of the common interferents toluene and o-xylene in ppm $_{\mathrm{v}}$-concentrations.
\end{abstract}

Key words: ion mobility spectrometry, high kinetic energy, benzene, toluene, humidity

\section{Introduction}

Benzene is not only an important chemical in the production of polymers, resins, adhesives, etc., but can be also released from natural sources such as fires and crude oil. Thus, it can be found in both indoor and outdoor environments. Benzene is therefore an important factor in air quality and work safety, as it has been classified by the International Agency for Research on Cancer (IARC) as carcinogenic to humans (Group 1) [1]. Typical legal exposure limits are in the range of $500 \mathrm{ppb}_{\mathrm{v}}$ to $1000 \mathrm{ppb}_{\mathrm{v}}$. However, it is of course desirable and prudent to monitor and avoid concentrations significantly below this maximum exposure. Therefore, measuring benzene in air samples at concentrations in the low ppb $_{\mathrm{v}}$-range and in real time is an important task. A variety of different sensors has been applied to this task, but typically they are either too complex for widespread field deployment, for example proton transfer reaction mass spectrometry (PTR-MS), or can only reach the required limits of detection using preconcentration, resulting in a too slow response, for example infrared absorption.

\section{Benzene Detection using IMS}

Extremely sensitive devices for fast trace gas detection in air are ion mobility spectrometers [2], often providing limits of detection in the low pptv-range for measurement times below one second. The basic operating principle of an ion mobility spectrometer with atmospheric pressure chemical ionization (APCI) is illustrated in Fig. 1. So-called reactant ions, typically protonated water clusters, are generated by means of a primary ionization source such as a radioactive or non-radioactive electron emitter or a corona discharge. These reactant ions undergo chemical gas-phase reactions with analyte molecules inside the ionization region, leading to the formation of analyte ions. This ionization process is both soft and extremely efficient, leading to the excellent limits of detection provided by ion mobility spectrometers. The ions thus generated are then injected into the drift region, where an electric field accelerates them towards a faraday plate detector. At the same time, the ions are slowed down by collisions with neutral molecules, causing each ion species to drift with an average speed proportional to the applied electric field and its ion mobility $K$. The ion mobility depends both on the experimental conditions and on the mass and size of the ion. 


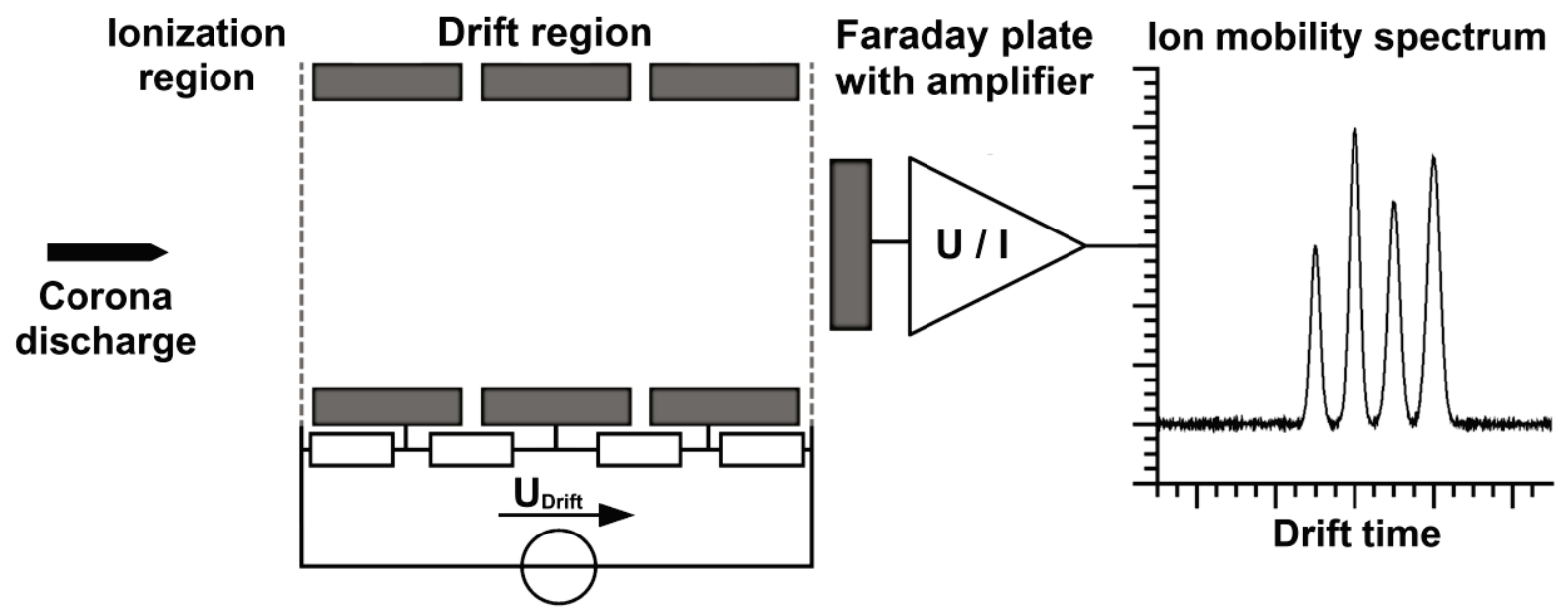

Fig. 1: Schematic setup of a drift tube ion mobility spectrometer utilizing a corona discharge source.

This way, different analyte ions are both separated from each other and from the initial reactant ions. The resulting ion mobility spectrum is, for drift tube or time-of-flight instruments, typically a plot of ion current over arrival time. From their characteristic peaks in this spectrum, different analytes can be separated and identified. The sensitivity and speed of this technique would in theory easily solve the measurement task at hand. In practical applications however, benzene provides several challenges regarding its detection by ion mobility spectrometry. First, the mobility of benzene ions is similar to both the mobility of the ubiquitous reactant ions from the atmospheric pressure chemical ionization process and the ions formed by the typical interferents toluene and xylene. This leads to overlapping peaks in the ion mobility spectrum, complicating the identification of the benzene peak in low resolution devices. This problem however is minor compared to the problems encountered during the ionization process. On the one hand, benzene has a proton affinity which is below the proton affinity of any water cluster larger than 1 , which is $\left(\mathrm{H}_{2} \mathrm{O}\right)_{n} \mathrm{H}^{+}$with $n>1$. Thus, it cannot be ionized by protonation under ambient conditions. The typical reaction pathway can therefore be expected to be charge transfer from other reactant ions, such as $\mathrm{NO}^{+}$. However, even a small concentration of water in the sample gas is sufficient to cause the formation of protonated water clusters from $\mathrm{NO}^{+}$, thus inhibiting this reaction pathway. Therefore, the sensitivity of ion mobility spectrometers for benzene is vastly reduced in humid samples. On the other hand, even if benzene has been ionized by one of the possible reactions, its ionization energy is rather high, especially compared to the common interferents toluene and xylene. Thus, even when benzene ions have been formed, there are likely to be quickly converted to other analyte ions, thus quenching the benzene signal. Again, this results in a vastly reduced sensitivity.

In order to overcome these problems, several modifications as listed in Tab. 1 may be made to the standard ion mobility spectrometer setup using atmospheric pressure chemical ionization. However, none of them are able to solve all the problems on their own and nearly all of them introduce new disadvantages. For example, ultraviolet light (UV) ionization sources directly ionize analyte molecules with sufficiently low ionization energy, thus eliminating the reactant ions and both the problems associated with peak overlap and the lack of suitable reaction pathways.

Tab. 1: Different approaches to facilitate benzene detection in ion mobility spectrometers.

\begin{tabular}{|c|c|c|c|c|}
\hline Technique & Peak overlap? & Humidity? & Interferents? & Disadvantages \\
\hline UV ionization & (Solved) & Solved & - & Reduced Sensitivity \\
\hline $\begin{array}{c}\text { GC / MCC } \\
\text { preseparation }\end{array}$ & - & (Solved) & Solved & $\begin{array}{c}\text { Delayed response, } \\
\text { reduced sensitivity }\end{array}$ \\
\hline Membrane inlet & - & Solved & - & Delayed response \\
\hline High resolution IMS & Solved & - & - & - \\
\hline
\end{tabular}




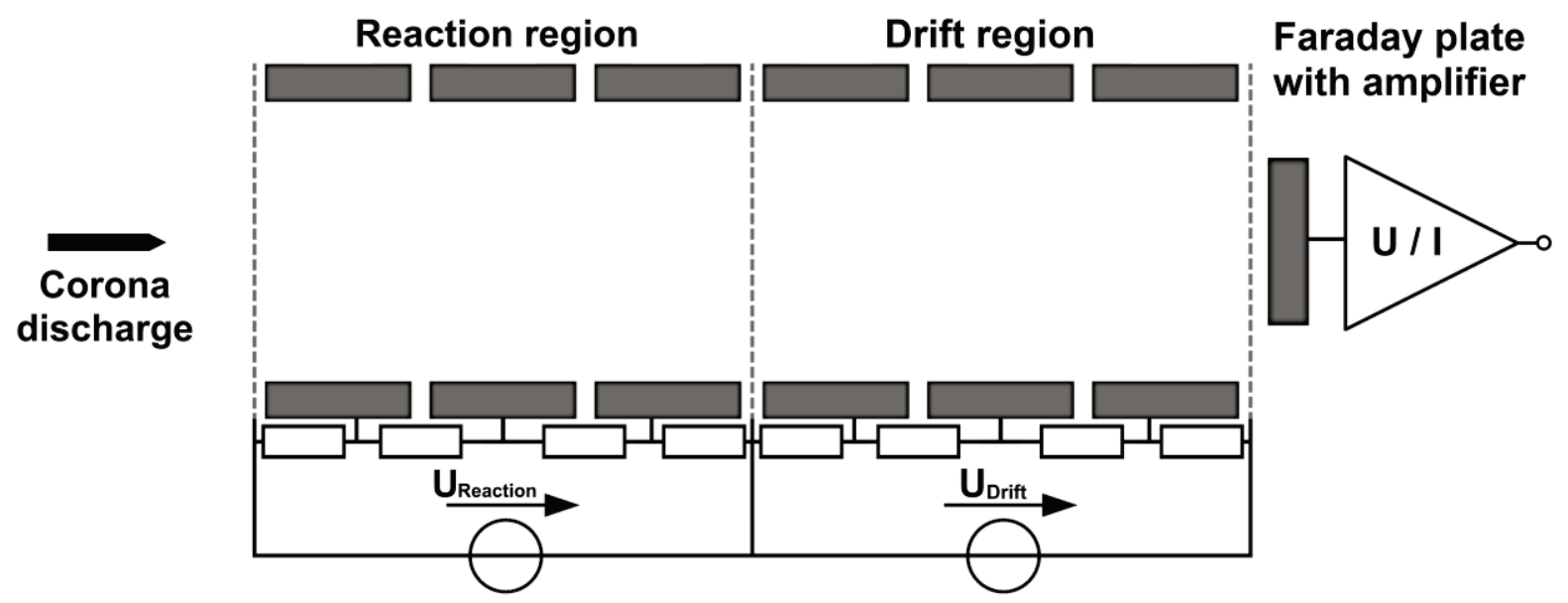

Fig. 2: Schematic setup of a high kinetic energy ion mobility spectrometer (Hike-IMS).

However, interferents may still cause peak quenching and peak overlap. Furthermore, as the ionization no longer occurs by a chemical gas phase reaction, UV ionization devices are typically significantly less sensitive than those utilizing atmospheric pressure chemical ionization.

Another possible improvement is the use of a preseparation, for example by gas chromatography (GC). A gas chromatograph can both separate humidity and interfering compounds from the benzene, thus mitigating the problems arising from analyzing mixtures. However, separation of the benzene peak from the reactant ion peak is still required inside the ion mobility spectrometer. Furthermore, a sufficient amount of time has to pass after the elution of water from the column, allowing the ion mobility spectrometer to dry before the elution of the benzene peak. Furthermore, the additional separation achieved by the gas chromatograph comes at a high price. The response of the system is delayed by the time the chemicals require to pass through the column, typically a few minutes, and as the signal is only present for a short period of time, only a few spectra of interest can be acquired during each run. Hence, the number of possible averages is significantly reduced, leading to increased noise and therefore increased limits of detection.

Typically, the preferred solution for the detection of benzene by ion mobility spectrometry is combining these techniques, resulting in a so-called GC-UV-IMS. However, while this device is well capable of the detection of benzene, it sacrifices the two most important advantages of trace gas detection by ion mobility spectrometry - sensitivity and speed. It should be noted that these problems are not necessarily limited to the detection of benzene, but apply to practically all low proton affinity / high ionization energy compounds when occurring in mixtures. Therefore, a general solution to these problems would be highly desirable to further the utility of ion mobility spectrometers.

\section{High Kinetic Energy IMS}

When analyzing the problems encountered during atmospheric pressure chemical ionization and the additional disadvantages introduced by the typical solutions, one general observation can be made: The atmospheric pressure chemical ionization is simultaneously one of the major strengths and one of the major weaknesses of ion mobility spectrometers, as it allows for their excellent sensitivity and speed, but its non-idealities also cause many problems in the applications. Solutions which try to solve these problems by foregoing the chemical ionization process therefore also always sacrifice the advantages associated with it. Thus, the most favorable solution to this dilemma would be to influence the chemical ionization in a way that preserves its advantages but mitigates it disadvantages.

This manipulation of the ionization process is the goal of the high kinetic energy ion mobility spectrometry (HiKE-IMS) [3], [4] as shown in Fig. 2. It utilizes an additional reaction region, similar to the reaction tubes used in proton transfer reaction mass spectrometry (PTR-MS) or selected ion flow tube mass spectrometry (SIFT-MS), to conduct the chemical ionization under defined conditions for a limited period of time (e.g. $100 \mu \mathrm{s})$. Typically, these conditions include reduced pressure (e.g. 20 mbar) in order to achieve a high reduced electric field strength. The reduced field strength is the ratio between the electric field strength $E$ and the number density of gas molecules $N$ and is measured in Townsend (Td). 


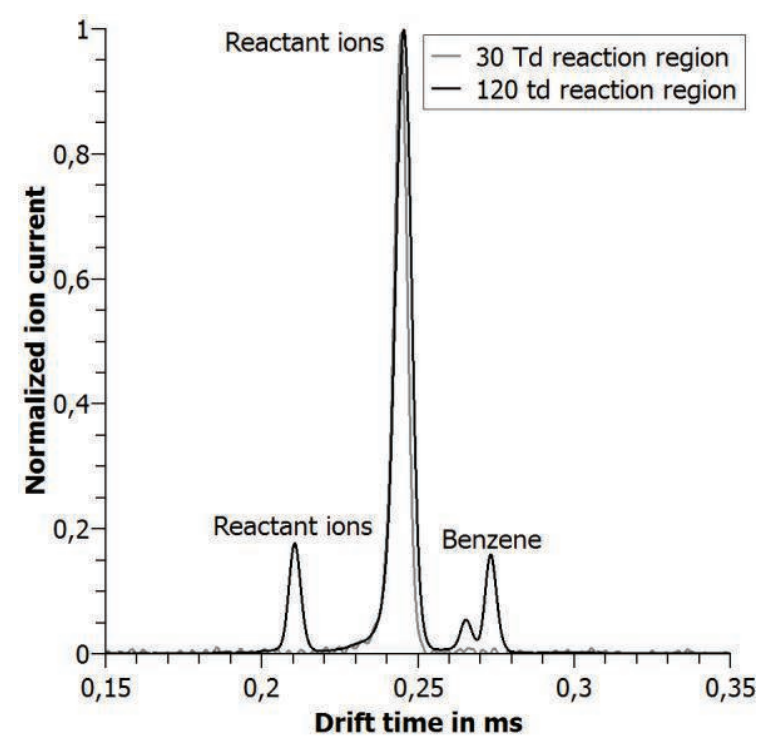

Fig. 3: Influence of the reduced electric field strength in the reaction region on the ionization of benzene.

At high reduced field strengths, ions obtain high kinetic energies, which lead to the fragmentation of the water clusters mentioned above. This reduces their proton affinity, thus opening new reaction pathways for the atmospheric pressure chemical ionization, which is illustrated in Fig. 3. It should be noted that this spectrum was acquired using also a high reduced electric field strength inside the drift region, which alters the ion mobility of different ions. This has also become possible due to the decreased pressure inside the high kinetic ion energy ion mobility spectrometer and mitigates the problems associated with peak overlap. When the reaction region is operated at a reduced electric field strength of $30 \mathrm{Td}$, which is already about ten to fifteen times higher than typical reduced field strengths in ion mobility spectrometry, no benzene peaks are visible. However, when the reduced electric field strength is increased to $120 \mathrm{Td}$, two distinctive benzene peaks form. One of them is created by charge transfer from $\mathrm{NO}^{+}$, the other by protonation from a water cluster.

The ability of the high kinetic energy ion mobility spectrometer to allow these reactions to occur even under adverse conditions is illustrated by the limits of detection in Fig. 4. In a dry sample gas, the device achieves a limit of detection of $8 \mathrm{ppb}_{v}$ for an averaging time of only $2 \mathrm{~s}$. However, when the humidity is increased to $3700 \mathrm{ppm}_{\mathrm{v}}$, which is more than sufficient to eliminate the formation of benzene ions in atmospheric pressure chemical ionization, the limit of detection only increases to $12 \mathrm{ppb}_{v}$ for the same averaging time. Thus, two of the three presented problems concerning the detection of benzene by ion mobility spectrometers could be solved by the use of high reduced electric field strength at reduced pressure.

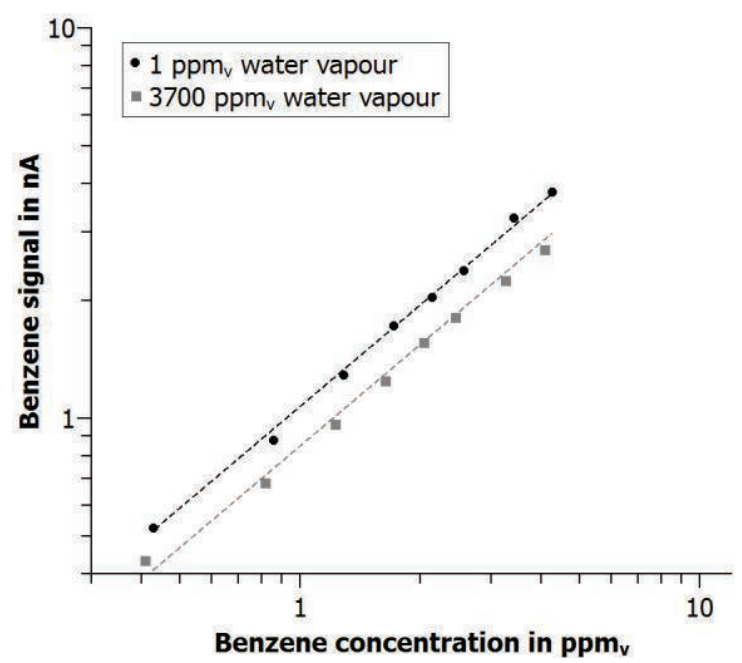

Fig. 4: Influence of humidity in the sample gas on the benzene signal.

However, the third problem still persists, which is the quenching of the benzene peak in the presence of interferents with higher proton affinity and lower ionization energy. To address this problem, high reactant ion densities are employed in combination with only a limited reaction time of about $100 \mu \mathrm{s}$. Thus, the ionization reaction does not reach its steady state yet and enough charge is available for the ionization of all analytes present in the sample, which is illustrated by Fig. 5. Typically, even toluene concentrations which are orders of magnitude lower than the present benzene concentrations are able to quench the benzene signal completely during atmospheric pressure chemical ionization.

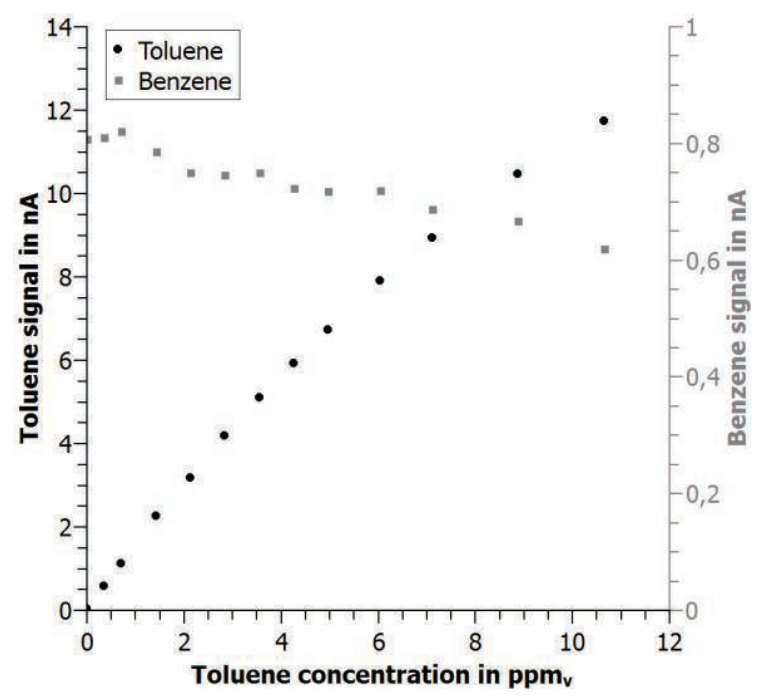

Fig. 5: Influence of toluene in the sample gas on the benzene signal. 


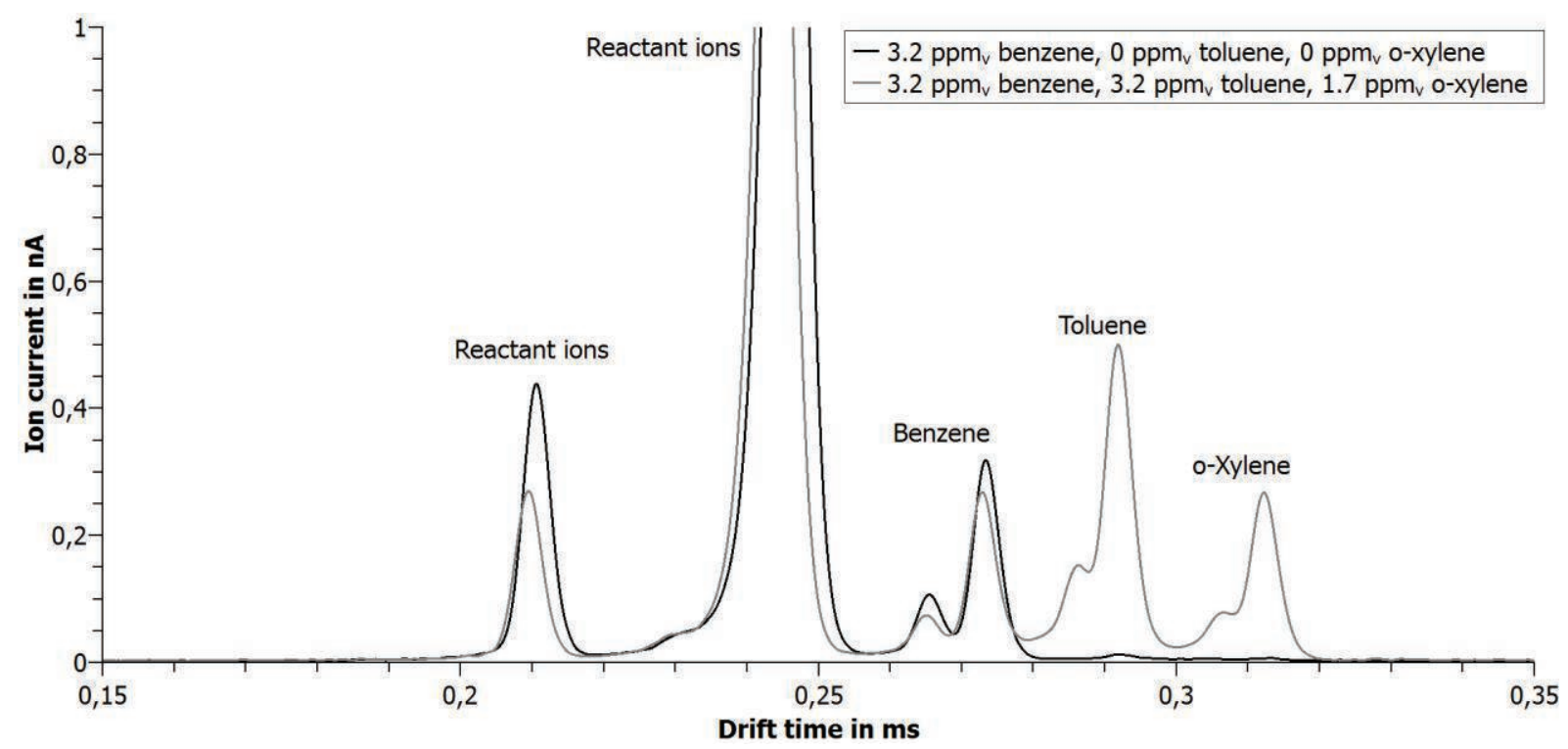

Fig. 6: Spectrum of benzene without and with the presence of interferents.

However, in the high kinetic energy ion mobility spectrometer, a benzene concentration of 850 $\mathrm{ppb}_{v}$ is still easily detectable even when 12.4 $\mathrm{ppm}_{\mathrm{v}}$ toluene are added to the sample gas. In fact, the benzene peak intensity only drops by about $25 \%$ despite the presence of a powerful interferent in significantly higher concentration. Overall, a limit of detection of $70 \mathrm{ppb}_{v}$ benzene for two seconds averaging time even under humid conditions $(90 \%$ r.H. $)$ and in the presence of toluene and o-xylene in $\mathrm{ppm}_{\mathrm{v}}$ concentrations has been achieved.

Thus, the problems described above concerning atmospheric pressure chemical ionization ion mobility spectrometers could be successfully mitigated by the use of the high kinetic ion energy ion source. It should be noted though that the operation of the ion mobility spectrometer at reduced pressure also reduces the general sensitivity and resolution of the device. However, the loss of sensitivity is in most cases recovered by the better performance of the high kinetic energy ion source when it comes to the ionization of complex samples. Additionally, the ability to control the ionizations process allows to extract additional information about the analytes, increasing the separation power. Furthermore, there is now only a limited dependence of the benzene signal on drastic changes of the sample parameters, such as the presence of humidity and interferents. Thus, the measurement is not only sensitive under adverse conditions, but also quantitative in certain bounds. This is illustrated in Fig. 6, where the peaks of benzene are exhibiting a near constant amplitude both without and with the presence of toluene and o-xylene.

\section{Conclusion}

Ion mobility spectrometers are fast and sensitive sensors for trace gas detection, but suffer from the non-idealities of the typically employed atmospheric pressure chemical ionization when applied to certain mixtures. For example, benzene becomes extremely difficult to detect when humidity or the common interferents toluene or xylene are present. By using a high kinetic ion energy source, these problems could be mitigated, allowing for fast, sensitive and even quantitative detection of benzene in the presence of interferents.

\section{References}

[1] IARC Monogr. Eval. Carcinog. Risks Hum., 7 (Suppl.), 1-440 (1987)

[2] G.A. Eiceman, Z. Karpas, H.H. Hill Jr., Ion Mobility Spectrometry, $3^{\text {rd }}$ Edition, CRC Press (2014)

[3] J. Langejuergen, M. Allers, J. Oermann, A. Kirk, S. Zimmermann, High Kinetic Energy lon Mobility Spectrometer: Quantitative Analysis of Gas Mixtures with Ion Mobility Spectrometry, Analytical Chemistry 86, 7023-7032 (2014); doi: 10.1021/ac5011662

[4] J. Langejuergen, M. Allers, J. Oermann, A. Kirk, S. Zimmermann, Quantitative Detection of Benzene in Toluene- and Xylene-Rich Atmospheres Using High-Kinetic-Energy lon Mobility Spectrometry (IMS), Analytical Chemistry 86, 11841-11846 (2014); doi: 10.1021/ac5034243 